\title{
Médiévales
}

Langues, Textes, Histoire

58 | printemps 2010

Humanisme et découvertes géographiques

\section{De la Grèce antique au voyage de Magellan. Les modèles humanistes d'Antonio Pigafetta et de Maximilianus Transylvanus}

From Ancient Greece to Magellan's Travel: Humanistic Models of Antonio

Pigafetta and Maximilianus Transylvanus

\section{Emmanuelle Vagnon}

\section{OpenEdition \\ Journals}

Édition électronique

URL : https://journals.openedition.org/medievales/5913

DOI : $10.4000 /$ medievales. 5913

ISSN : $1777-5892$

\section{Éditeur}

Presses universitaires de Vincennes

\section{Édition imprimée}

Date de publication : 30 juin 2010

Pagination : 99-111

ISBN : 978-2-84292-260-3

ISSN : 0751-2708

\section{Référence électronique}

Emmanuelle Vagnon, « De la Grèce antique au voyage de Magellan. Les modèles humanistes d'Antonio Pigafetta et de Maximilianus Transylvanus », Médiévales [En ligne], 58 | printemps 2010, mis en ligne le 20 septembre 2012, consulté le 22 avril 2022. URL : http://journals.openedition.org/ medievales/5913; DOI : https://doi.org/10.4000/medievales.5913 
Médiévales 58, printemps 2010, p. 99-111

Emmanuelle VAGNON

\section{DE LA GRÈCE ANTIQUE AU VOYAGE DE MAGELLAN. LES MODÈLES HUMANISTES D'ANTONIO PIGAFETTA ET DE MAXIMILIANUS TRANSYLVANUS}

Le voyage de Fernand de Magellan et de ses marins est une étape majeure dans l'histoire des Grandes Découvertes. Première circumnavigation du globe, par le sud du continent américain, il avait pour objectif de trouver un passage vers l'Asie des épices en voyageant vers l'ouest, dans un contexte de rivalité commerciale et politique entre le Portugal et l'Espagne. En effet, les deux couronnes s'étaient partagé le monde au moment du traité de Tordesillas : l'hémisphère oriental revenait au Portugal, l'hémisphère occidental à l'Espagne'. Il s'agissait pour Magellan de rejoindre l'archipel des Moluques, sans traverser l'espace commercial des Portugais, et de trouver l'emplacement exact de l'antiméridien de Tordesillas, de l'autre côté du globe. Le voyage fut mouvementé ${ }^{2}$. Une mutinerie éclata, puis l'équipage du San Antonio, découragé, déserta et le navire retourna vers l'Espagne avant le passage du détroit vers le Pacifique. Magellan lui-même n'acheva pas le tour du monde : il mourut en chemin lors d'une bataille contre des indigènes. Quelques-uns de ses compagnons seulement effectuèrent la première circumnavigation complète, sous le commandement de Juan Sebastián Elcano, et rapportèrent avec eux le récit de cet exploit ${ }^{3}$. Le plus célèbre de ces témoignages, celui aussi

1. Pour les commentaires sur les aspects historiques de l'expédition, $c f$. X. DE CASTro, J. Hamon, L. F. Thomaz, Le Voyage de Magellan (1519-1522). La relation d'Antonio Pigafetta et autres témoignages, Paris, 2007, notamment la préface, p. 7-32 et les nombreuses notes. Le traité de Tordesillas du 7 juin 1494 fixait la frontière à 370 lieues à l'ouest des îles du Cap Vert. L'expédition de Vasco de Gama de 1497 permit d'établir la route des Indes au-delà du Cap de Bonne Espérance et d'étendre le domaine des Portugais vers l'Océan Indien puis l'Indonésie. La carte dite « de Cantino » de 1502 est la première à représenter le «méridien de Tordesillas ».

2. Au départ de l'expédition, 237 hommes répartis sur cinq navires : la Trinidad, la Victoria, le San Antonio, la Concepcion et le Santiago (détruit en route).

3. Magellan mourut dans l'île de Mactan, le 27 avril 1521. La Victoria regagna l'Espagne à Sanlúcar de Barrameda le 6 septembre 1522. 
qui est considéré en général comme le plus complet et le plus proche des faits ${ }^{4}$, est celui d'Antonio Pigafetta, connu par plusieurs manuscrits illustrés, et intitulé Navigation \& découvrement de l'Inde supérieure \& îles de Malucque où naissent les clous de girofle, faite par Antonio Pigafetta, vicentin et chevalier de Rhodes, commençant en l'an $1519^{5}$.

La forme utilisée par l'auteur pour diffuser son récit n'est pas anodine. Les manuscrits semblent entrer dans la catégorie de l'isolario ou insulaire, un genre littéraire et cartographique particulier, décrivant les îles du monde selon un itinéraire maritime réel ou fictif, ou bien comme un catalogue encyclopédique, et toujours illustré de cartes plus ou moins élaborées ${ }^{6}$. Cette disposition a été adoptée à l'origine par le prêtre florentin Cristoforo Buondelmonti dans le Liber insularum Archipelagi, ouvrage rédigé dans les premières décennies du $\mathrm{XV}^{\mathrm{e}}$ siècle, et qui combine une description des îles et des habitants de la mer Égée de son époque avec des commentaires humanistes sur la mythologie et l'histoire antiques ${ }^{7}$. On peut donc se demander en quoi l'œuvre de Pigafetta s'apparente à ce genre cartographique pour représenter et mettre en valeur les terres jusque-là inconnues. Jusqu'à quel point Antonio Pigafetta suit-il des modèles humanistes et comment la redécouverte archéologique du monde antique de Cristoforo Buondelmonti pouvait-elle être transposée à la découverte de mondes radicalement nouveaux ?

Or l'exploit de Magellan a été raconté également par d'autres témoins. Pour mettre en évidence les choix de Pigafetta, il faut les comparer aux autres

4. X. DE CASTRO, op. cit., p. 67-71: mise au point historiographique.

5. Plusieurs éditions récentes sont venues renouveler et corriger l'ouvrage vieilli de Léonce Peillard. Antonio Pigafetta, Relazione del primo viaggio attorno al mondo, A. Canova éd., Padoue, 1999 ; ID., The First Voyage Around the World (1519-1522). An account of Magellan's Expedition, T. J. Cachey éd., Toronto, 2007 ; X. De Castro, J. Hamon, L. F. Thomaz, Le Voyage de Magellan (1519-1522), op. cit., t. 1, p. 77-261.

6. Pour une définition et un historique des insulaires, $c f$. F. Lestringant, Le Livre des îles. Atlas et récits insulaires de la Genèse à Jules Verne, Genève, 2002 ; G. Tolias, « Isolarii, Fifteenth to Seventeenth Centuries », dans D. Woodward dir., The History of Cartography, vol. 3, ChicagoLondres, 2007, chapitre 8.

7. Cristoforo Buondelmonti, Christophori Bondelmontii Florentini Liber insularum Archipelagi, G. R. L. DE Sinner éd., Leipzig-Berlin, 1824 ; ID., Description des îles de l'Archipel, E. Legrand éd., Paris, 1897 ; ID., Descriptio insule Crete et Liber insularum, M.-A. VAN SPITAËL éd., Héracleion, 1981. L'étude de Claudia Barsanti est la meilleure synthèse actuelle sur l'ensemble de l'œuvre de Buondelmonti : C. BARSANTI, «Costantinopoli e l'Egeo nei primi decenni del Xv secolo : la testimonianza di Cristoforo Buondelmonti », Rivista dell'Istituto Nazionale d'Archeologia e Storia dell'Arte, t. 56 (III Serie, xxIv), 2001, p. 83-253 ; G. RaGone, «Il Liber insularum Archipelagi di Cristoforo dei Buondelmonti : filologia del testo, filologia dell'immagine », dans D. MARCotTE dir., Humanisme et culture géographique à l'époque du concile de Constance, Actes du colloque de l'Université de Reims, 18-19 novembre 1999, Turnhout, 2002, p. 177-217. 
récits, aisément disponibles dans une édition récente ${ }^{8}$. Mises à part les dépositions des marins déserteurs, interrogés à leur retour par les autorités espagnoles et portugaises ${ }^{9}$, les témoignages ont tous été soigneusement élaborés. C'est le cas par exemple de la lettre rédigée par un humaniste et pour un public humaniste : le récit de Maximilianus Transylvanus, secrétaire de Charles Quint, daté de septembre 1522 et imprimé en 1523, avant même la diffusion du récit de Pigafetta ${ }^{10}$.

À travers ces deux exemples, nous voulons montrer à quel point les récits de l'un des épisodes les plus remarquables des Grandes Découvertes s'inscrivent dans un rapport ambigu de rupture mais aussi d'hommage par rapport au savoir humaniste, tout en requérant les supports éditoriaux conformes aux goûts des cercles cultivés de l'époque.

\section{Pigafetta auteur d'un nouveau Livre des îles}

On sait peu de choses sur Antonio Pigafetta avant son aventure maritime. Issu d'une noble famille de Vicence, il accompagne en Espagne le nonce apostolique Chiericati en tant que secrétaire. Il embarque en 1519 à bord de la Trinidad dans l'équipage de Fernand de Magellan. Il fait partie des marins survivants arrivés les premiers en Espagne et remet dès son retour une copie de son journal de bord à Charles Quint. Il se rend ensuite à Lisbonne pour rencontrer Jean III de Portugal et en France auprès de la mère du roi François ${ }^{\text {er }}$, Louise de Savoie, à qui il offre également un exemplaire de son œuvre ${ }^{11}$. Ces premières versions du texte, les plus proches des notes prises au cours du voyage, sont aujourd'hui perdues ${ }^{12}$. Puis, de retour en Italie début 1523 , il continue ou remanie son texte pour le diffuser plus largement. Il cherche alors la protection du duc de Mantoue, puis du pape Clément VII, et s'adresse enfin au grand maître de l'ordre de Rhodes Philippe de Villiers de l'Isle Adam, alors réfugié à Monterosi, près de Viterbe (août 1524). L'intérêt de ce dernier lui vaut de devenir lui-même chevalier de Rhodes en 1524 et de recevoir probablement une pension ${ }^{13}$. Les manuscrits conservés reflètent la version

8. X. De Castro, J. Hamon, L. F. Thomaz, op. cit. .

9. X. DE CASTRO, op. cit., p. 555-596.

10. Ibid., p. 883-918.

11. Comme il le raconte lui-même à la fin de son récit, $c f$. X. DE CASTro, op. cit., p. 261.

12. Il existe cependant une traduction résumée de ces notes par Jacques-Antoine Fabre, publiée entre 1526 et 1536. Cf. X. DE CASTro, op. cit., p. 1018.

13. Dédicace des manuscrits en français : «Antonio Pigafetta, patricien vicentin et chevalier de Rhodes, à l'illustrissime et très excellent seigneur Philippe de Villiers de l'Isle-Adam inclite grand maître de Rhodes et son seigneur observantissime ». Le prologue évoque aussi la protection de ce personnage, $c f$. X. DE CASTRO, op. cit., p. 81 ; TH. J. CACHEY Jr, « Italy and the invention of America », New Centennial Review, vol. 2, 1, 2002, p. 17-31, en particulier p. 27. 
du texte dédicacée à Philippe de Villiers de l'Isle Adam. Ils ont tous une présentation très soignée. Trois sont en français, tandis que le quatrième, en italien, daté de 1525 , est considéré comme le plus complet ${ }^{14}$. Puis, entre 1526 et 1536, une version abrégée en français, et des versions en italien et en anglais, sont imprimées et largement diffusées ${ }^{15}$. Ces éléments biographiques montrent que le voyage n'a pas apporté immédiatement à Pigafetta la richesse et la gloire qu'il recherchait ${ }^{16}$. Pour se faire entendre, et faire valoir l'expérience exceptionnelle qu'il venait de vivre, il a dû à son retour obtenir la reconnaissance des cercles du pouvoir, et trouver des subventions pour assurer son existence. Un des moyens d'attirer l'attention était de présenter son témoignage de la manière la plus favorable et de le faire publier largement.

Le récit de Pigafetta se présente comme un journal du voyage d'exploration maritime, où sont relatés les principaux événements. Le texte contient également des indications nautiques : caps, largeur des baies et détroits, profondeurs, direction des vents. Néanmoins, les détails techniques d'un véritable journal de bord ont été effacés au profit du récit ; quand ils sont maintenus, ils sont parfois expliqués au lecteur non averti ${ }^{17}$. Par ailleurs, des remarques sur la nouveauté du passage vers le Pacifique, ainsi que quelques notions de cosmographie classique, soulignent que Pigafetta est conscient de l'enjeu de l'expédition et de l'exploit que les marins sont en train d'accomplir ${ }^{18}$. Certaines de ces remarques ont peut-être été rajoutées lors du remaniement du

14. $A=$ BnF, ms. fr. 5650 ; B = BnF, ms. fr. 24224 ; $C=$ Yale University, Beinecke Library, ms. 351 ; D = Milan, Biblioteca Ambrosiana, ms. L 103 Sup. A, C et D ont une source commune italienne, aujourd'hui perdue. Le ms. B est une copie incomplète de A.

15. Le récit est repris en italien par Giovanni Battista Ramusio dans ses Navigationi et Viaggi (Venise, 1550-1559, réédité plusieurs fois au XVI ${ }^{\mathrm{e}}$ siècle). Dernière édition de Marica Milanesi, Turin, 1978-1988, volume II, p. 831-955.

16. X. DE CASTro, op. cit., p. 81 : «j'ai fait ledit voyage et bien vu à l'œil les choses ci-après écrites, et pour m'acquérir quelque fameux nom après la postérité ».

17. Par exemple, l'auteur prend la peine d'expliquer le terme «garbin »: «Après que nous eûmes passé la ligne équinoxiale vers le midi, nous perdîmes l'étoile de la tramontane, naviguâmes entre le vent de midi et le garbin, qui est le vent collatéral entre ledit midi et le ponant, et traversâmes jusqu'à une terre nommée Verzin, qui est en $24^{\circ} 30^{\prime}$ du ciel Antarctique » (X. de Castro, op. cit., p. 89).

18. Exemple, X. DE CASTro, op. cit., p. 117 (13 février 1521) : «Durant ce temps de deux mois et douze jours, nous naviguâmes entre le ponant et le maestral, et à la quarte du maestral vers le ponant, et au maestral jusqu'à ce que nous vînmes à la ligne équinoxiale, qui est loin de la ligne de répartition de $122^{\circ}$. Laquelle ligne de répartition est loin de $30^{\circ}$ du premier méridien, lequel est loin du Cap Vert de $3^{\circ}$ au Levant [...] pour nous approcher à la terre du cap de Gaticara, lequel cap (sous correction de ceux qui ont fait la cosmographie, car ils ne l'ont point vu) ne se trouve pas où ils pensent, mais est vers le septentrion, à $12^{\circ}$ environ ». 
texte, de même que le Traité de la Sphère, situé à la fin de certains manuscrits, et qui donne une caution scientifique au livre ${ }^{19}$.

On sait en effet que Pigafetta a complété son texte après son retour en utilisant des descriptions géographiques plus anciennes ${ }^{20}$. Mais l'œuvre de Cristoforo Buondelmonti n'a pas été utilisée directement, même si Pigafetta semble s'inscrire dans une même tradition de littérature géographique. C'est ainsi qu'on ne verra pas dans l'œuvre de Pigafetta l'érudition dont témoigne le Liber insularum de Cristoforo Buondelmonti, où chaque île grecque est surtout un prétexte à un souvenir tiré de la littérature latine, en particulier de la lecture de Virgile. Certes, point de vestiges antiques dans les îles du Nouveau Monde : en revanche, Pigafetta laisse une place importante aux descriptions de la faune, de la flore et des habitants. C'est en cela que l'on peut retrouver des points communs avec Buondelmonti, et pour cet aspect seulement de la culture géographique humaniste : l'intérêt pour les ressources naturelles des îles. En effet, le texte du voyageur florentin du $\mathrm{XV}^{\mathrm{e}}$ siècle contient des remarques authentiques sur l'état contemporain de l'Archipel grec, à l'époque où l'avancée ottomane menaçait les colonies latines de la mer Égée. La production agricole, la qualité des sols, l'emplacement des îles sont ainsi décrits dans une perspective géopolitique qui se perçoit plus nettement dans les versions longues de l'œuvre de Buondelmonti, ou dans sa traduction tardive en italien, largement remaniée ${ }^{21}$. Deux lectures ou deux usages du Livre des îles de Buondelmonti en ont donc fait le succès : d'une part comme manuel humaniste favorisant le voyage en imagination à travers la littérature antique, rappelant en cela 1'Itinerarium ad sepulcrum Domini nostri Ihesu Cristi de Pétrarque ${ }^{22}$; d'autre part, comme témoignage géographique et politique à l'usage des

19. Ce traité est cité dans le récit aux côtés d'un passage d'Aristote, et sur le même plan que ce dernier : « Nous entrâmes audit port (Rio de Janeiro) le jour de sainte Lucie, ès avents Noël, auquel jour nous eûmes le soleil par zénith, qui est un terme d'astrologie. Ce zénith est un point du ciel qui, selon les astrologiens, et seulement à l'imagination, répond sur notre tête par droite ligne, comme il peut se voir par le Traité de la Sphère et par Aristote, au premier livre de Celo \& Mondo ». (13 nov. 1519, X. DE CASTRO, op. cit., p. 90.) Seuls les manuscrits A et D contiennent le Traité de la Sphère. Il s'agirait d'une copie confuse du Tratado de la longitud ó altura de leste oeste, par Rui Faleiro. Cf. X. DE CASTRO, op. cit., p. 348, n. 2.

20. Entre autres, l'œuvre de Niccolò de' Conti, $c f$. X. DE CASTro, op. cit., p. 72.

21. La version tardive du Liber insularum, datée de 1430, est connue par un seul manuscrit écrit dans un dialecte des Marches (Vatican, BAV, Ross. 704). Elle a été fortement remaniée avec des passages postérieurs à 1470 .

22. CEuvre de Pétrarque datée de mars 1358. Cf. F. Lo Monaco, Itinerario in Terra santa, 1358, Bergame, 1990 et «Itinerarium ad sepulchrum domini nostri Yehsu Christi », Petrarch's guide to the Holy land. Facsimile edition of Cremona, Biblioteca Statale, Deposito Libreria Civica, manuscript BB.1.2.5., TH. J. CACHEY éd., Notre Dame (Indiana), 2002. 
navigateurs et des commerçants ${ }^{23}$. Or c'est bien ce dernier usage qui est privilégié par Pigafetta lorsqu'il introduit des remarques ethnographiques reconnues pour leur authenticité ${ }^{24}$, et lorsqu'il manifeste un intérêt prononcé pour la langue des peuples rencontrés (un lexique est inséré dans le récit). À peine une certaine place est-elle laissée au merveilleux, comme dans une tradition littéraire du récit de voyage et de description géographique, qui remonte à Pline et Solin. Ainsi, dans un fameux passage sur les géants de Patagonie : « et il était tant grand que le plus grand de nous ne lui venait qu'à la ceinture ${ }^{25} »$.

D'autre part, c'est surtout par son illustration que la Navigation de Pigafetta rappelle les manuscrits du Liber insularum de Buondelmonti, comme le montre aisément la comparaison de plusieurs « figures d'île ${ }^{26}$ ». Des cartes sommaires, représentant les principales étapes du parcours des navires de Magellan, sont encadrées pour les isoler du texte et le nom des îles est inscrit sur des banderoles. Les îles sont souvent regroupées ; leur forme est esquissée sans échelle, ni indication de distances ou de durées de voyage, leur topographie est suggérée par des symboles cartographiques très simples pour figurer les reliefs, et plus rarement les cours d'eau. En revanche, à la différence des manuscrits de Buondelmonti, aucune occupation humaine n'est relevée, ni davantage la faune ni la flore, si bien que, du strict point de vue cartographique, ces croquis n'apportent pas grand-chose à la description des îles nouvelles et sont très pauvres en renseignements maritimes. Quelle est donc alors leur fonction?

23. Cette double destination est soulignée dans le prologue du manuscrit du Vatican, BAV, Ross. 704, $\mathrm{f}^{\circ} \mathrm{IV}$ : «Anchi mercanti ne reportarando fructo non pocho, per che ho trovato in queste isole cose miravigliose si de medicine ad salute dellu corpo humano come anchi ad altre cose, per che multi pensano quelle esser portate de India over longinqui paesi \& lochi [...] Serrà ancora utele assai alli naviganti, nochieri, pedoti et altri che vanno colli loro over alieni legni per che io depingo le isole insieme colli porti reducti e le sue conditioni. »

24. On peut se demander si l'insistance sur les mœurs sexuelles des peuples rencontrés ne relève pas autant de l'intérêt anthropologique pour une autre forme d'organisation naturelle et sociale des humains, que de l'anecdote destinée à attirer la curiosité des lecteurs. Certains de ces détails ont été supprimés d'un des manuscrits (Paris, BnF, ms. fr. 24224).

25. X. De CAStro, op. cit., p. 97. Cf. A. CAnova, «Esperienza e letteratura nella Relazione del viaggio attorno al mondo di Antonio Pigafetta : la descrizione del Brasile », Annali di storia moderna e contemporanea, t. 4, 1998, p. 459-476.

26. Pour les cartes des manuscrits de Buondelmonti, quelques exemples reproduits dans R. Almagì̀, Planisferi e Carte nautiche affini dal secolo XIV al XVII esistenti nella Biblioteca Vaticana (Monumenta Cartografica Vaticana, vol. I), Cité du Vatican, 1944, p. 105-117 ; C. Barsanti, loc. cit. L'intégralité des illustrations du manuscrit de Yale de l'œuvre de Pigafetta est reproduite dans l'édition de X. DE CASTRo, op. cit. 


\section{La forme de l'insulaire : un choix éditorial}

Le choix du genre de l'isolario s'explique d'abord comme une tentative d'intégration des mondes nouveaux à l'espace connu. Comme une précédente journée d'étude a permis de le souligner ${ }^{27}$, l'île, qui fonctionne comme un microcosme, est le lieu idéal de la description anthropologique. Pigafetta décrirait ainsi le monde comme un immense archipel où chaque morceau d'humanité présente ses spécificités propres. La différence avec le Liber insularum Archipelagi tient dans le fait que « ces îles nouvelles sont sans villes et sans monuments, sans écriture et sans mémoire ${ }^{28} »$. Par ailleurs, comme d'autres auteurs à la même époque, Pigafetta cherche par ce moyen à rendre compte et à donner à voir une représentation, nécessairement incomplète, fragmentée, d'un monde en cours d'exploration.

Selon le spécialiste de la littérature de la Renaissance, Theodor J. Cachey, Pigafetta aurait surtout choisi ce type de mise en forme de son récit en référence à son destinataire et protecteur, le grand maître de l'ordre des chevaliers de Rhodes ${ }^{29}$. Il est vrai que c'est à Rhodes que Buondelmonti a écrit son récit et que plusieurs manuscrits ont été copiés ${ }^{30}$. Cependant, cette hypothèse laisse croire que le modèle de l'isolario serait typiquement lié à l'ordre de Rhodes et que le récit de Buondelmonti aurait eu une influence considérable auX $\mathrm{XV}^{\mathrm{e}}$ et $\mathrm{XVI}^{\mathrm{e}}$ siècles dans ce milieu précis. On peut émettre quelques doutes à ce sujet, au regard de l'histoire de la diffusion du Liber insularum Archipelagi, qui, certes, a été copié un très grand nombre de fois (plus de soixante manuscrits conservés, dans des versions différentes et de qualité très inégale), mais qui n'a jamais été imprimé. De plus, les historiens n'ont pas à ce jour trouvé trace d'une carrière remarquable du prêtre florentin au sein de l'ordre de Rhodes/Malte. En revanche, on peut souligner qu'à la même période, Piri Reis réalise pour Soliman le Magnifique son propre Livre pour naviguer (Kitab-i-Bahriye), à un moment décisif de l'expansion turque en mer Égée : une première version en 1521 , la deuxième

27. A. Franzini et N. Bouloux dir., Îles du Moyen Âge, Médiévales, $\mathrm{n}^{\circ}$ 47, automne 2004.

28. F. Lestringant, «La voie des îles », dans A. Franzini et N. Bouloux dir., op. cit., p. 113-122, en particulier p. 116-117.

29. Th. J. CACHEy Jr, «Italy and the invention of America », New Centennial Review, vol. 2, 1, 2002, p. 17-31, en particulier p. 26-28 ; TH. J. CACHEY Jr., « Maps and Literature in Renaissance Italy », dans D. WoodWARD dir., The History of Cartography, vol. 3, op. cit., p. 450-460, en particulier p. 459-460.

30. Comme nous le prouvent plusieurs dédicaces ou colophons de manuscrits. Ex. Paris, BnF, ms. latin 4823 : Incipit Liber insularum arcipelagi editus per presbyterum Christophorum de Bondelmontibus de Florentia quem misit de civitate Rhodi Romam domino Jordano Cardinali de Ursinis Anno Domini M CCCC XXII ; Vatican, BAV, Chigi F. IV. 74 : Sed ego, Honufrius de Penna ipsum librum cum alio anteriori copiavi et scripsi manu propria Rodi. 
en $1526^{31}$. En 1522, les troupes du sultan parviennent à chasser les chevaliers de Rhodes ; ceux-ci restèrent en exil jusqu'à leur installation dans l'île de Malte en 1530. L'œuvre de Piri Reis reflète ainsi l'intérêt du nouveau seigneur ottoman des îles grecques pour le genre de l'isolario, au moment même où le grand maître des chevaliers de Rhodes porte son attention sur Pigafetta et les îles du Nouveau Monde.

De fait, il convient de replacer les manuscrits de Pigafetta dans un courant éditorial qui se développe dans les premières décennies du $\mathrm{XVI}^{\mathrm{e}}$ siècle et qui dépasse l'horizon grec et italien. La production d'ouvrages cartographiques prestigieux, richement illustrés, avait pris une ampleur remarquable depuis la fin $\mathrm{du} \mathrm{XV}^{\mathrm{e}}$ siècle, et tout d'abord en Italie du Nord, à Venise et à Florence, puis dans d'autres pays au début du XVI ${ }^{\mathrm{e}}$ siècle. Le rapport entre les milieux de bibliophiles humanistes et les authentiques navigateurs est confirmé par la mode des atlas nautiques et livres de navigation richement ornés, cartes marines et insulaires, qui se multiplient alors ${ }^{32}$. Ces recueils de cartes, d'abord limités à la Méditerranée, furent ensuite étendus au reste du monde connu, en utilisant à la fois les sources antiques disponibles et les résultats des découvertes récentes ${ }^{33}$. À Florence, dans les années 1480-1490, le cartographe allemand Henricus Martellus réalise plusieurs ouvrages de luxe, dans lesquels il mêle la Géographie de Ptolémée, le Liber insularum Archipelagi, et le savoir récent des navigateurs et des cartographes. Au fil du temps, l'auteur propose une représentation modernisée et corrigée de la mappemonde ptoléméenne, en y faisant figurer certaines des nouvelles découvertes, notamment le Cap de Bonne Espérance et l'ouverture de l'Océan Indien ${ }^{34}$. La mise à jour des cartes existantes à l'aide d'informations récentes demeure un aspect fondamental de l'ensemble de son projet, comme le souligne le

31. S. SoucEK, « Islamic charting in the Mediterranean », dans J. B. HARLEY etD. WoOdWARD dir., The History of Cartography, vol. 2, t. 1, Chicago, 1992, p. 272-279.

32. F. X. Leduc et M. Pelletier, «Les insulaires », dans M. Pelletier dir., Couleurs de la Terre. Des mappemondes médiévales aux images satellitales, Paris, 1998, p. 56 ; F. LeSTRINGAnt, Le Livre des îles, op. cit. ; G. Tolias, « Isolarii, Fifteenth to Seventeenth Centuries », loc. cit.

33. Les cartes marines luxueuses produites dans la seconde moitié du $\mathrm{XV}^{\mathrm{e}}$ siècle par l'atelier de Grazioso Benincasa, originaire d'Ancône et actif à Venise, témoignent de cet enrichissement des cartes par l'apport des découvertes portugaises. Elles font partie des premières cartes à représenter le sud-ouest de la côte africaine et les îles du Cap Vert. Cf. E. VAGnoN, «Un Atlas retrouvé de Grazioso Benincasa. Cartographie marine à la fin du Xve siècle d'après les collections de la Bibliothèque nationale de France », Revue du Comité français de la Cartographie, t. 184, 2005, p. 12-22.

34. R. Almagià, « I mappamondi di Enrico Martello e alcuni concetti geografici di Cristoforo Colombo », La Bibliofilia, t. 42, 1940, p. 288-311 ; L. BöNINGER, Die deutsche Einwanderung nach Florenz im Spätmittelalter, Leyde-Boston, 2006, chapitre VII, « Arrigho di Federigho Martello; Bürgerknecht, Übersetzer und Kartograph », p. 313-354 ; A. O. VIETOR, « A Pre-Columbian map of the world, circa $1489 »$, Imago Mundi, t. 17, 1963, p. 95-96. 
titre d'un de ses manuscrits de la Géographie de Ptolémée ${ }^{35}$. L'intégration du Nouveau Monde à l'espace cartographique connu était donc déjà en cours lorsque Pigafetta proposa son récit illustré ${ }^{36}$.

Il est difficile néanmoins de savoir quel ouvrage a pu directement inspirer Pigafetta au moment où il fit réaliser les manuscrits de luxe destinés à des souverains et au grand maître de l'Ordre de Rhodes. Notons qu'un certain Valentim Fernandes, actif à Lisbonne au début du XVI ${ }^{\mathrm{e}}$ siècle, réalisa en 1507 un isolario qui rendait compte des découvertes portugaises. Il en fit parvenir une partie à l'humaniste allemand Konrad Peutinger, qui le publia sous le titre De insulis et peregrinationem Lusitanorum ${ }^{37}$. Bien que le style des cartes soit différent, il est possible que Pigafetta ait eu connaissance de cet insulaire. Bien plus, il serait intéressant d'étudier de près les ateliers de copistes disponibles en France et en Italie du Nord au début du XVI ${ }^{\mathrm{e}}$ siècle. Un manuscrit français méconnu, précieusement illustré de peintures raffinées, associe une traduction française de l'œuvre de Buondelmonti à un portulan des côtes atlantiques ${ }^{38}$. Par ailleurs, une véritable ressemblance stylistique se dégage entre les manuscrits réalisés en France pour illustrer l'œuvre de Pigafetta et certains exemplaires du Liber insularum de Buondelmonti, ce qui laisse supposer la facture d'un même atelier français spécialisé dans la production de manuscrits cartographiques au début du XVI ${ }^{\mathrm{e}}$ siècle, et auquel Pigafetta aurait fait appel ${ }^{39}$.

L'hypothèse la plus probable est donc que Pigafetta suivait surtout un modèle éditorial, de diffusion de ce genre de littérature, destiné à un certain public. La forme comptait autant que le fond quand il s'agissait de plaire à un milieu littéraire avide de récits de voyage et friand de beaux manuscrits

35. Florence, Biblioteca Nazionale Centrale, Magl. Cl. XIII-16, f. 1v ${ }^{\circ}$ : Cl(audii) Ptolomei Cosmographia cum tabulis regionum nostri temporis et universis portobus (sic) et locis maritimi tractus tam notis quam a rege portusgalli nuper repertis hoc ornatissimo codice continentur.

36. D'autres jalons de l'histoire des insulaires sont bien connus. Citons pour exemple l'Isolario du Vénitien Bartolomeo dalli Sonetti (édité en 1484-85), le Libro di tutte le isole del mondo de Benedetto Bordone (1528).

37. Munich, Bayerische Staatsbibliothek, Codex monacensis hispanicus 27 ; ACADEMIA PORTUGUesa DA História, Codice Valentim Fernandes, Lisbonne, 1997. Ce personnage avait par ailleurs publié en 1502 l'œuvre de Nicolo de'Conti, dont on sait que Pigafetta a utilisé certains passages. $C f$. G. Seibert, « 500 Years of the Manuscript of Valentim Fernandes, a Moravian Book Printer in Lisbon », dans B. E. CIESZYŃsKa dir., Iberian and Slavonic Cultures: Contact and Comparison, Lisbonne, 2007, p. 79-88. Je remercie G. Tolias pour cette information ainsi que pour les références qui y sont associées.

38. Paris, BnF, ms. fr. 2794. Ce manuscrit aux miniatures très fines, daté entre 1504 et 1515 , porte les armes de François de Valois, comte d'Angoulême, futur François ${ }^{\text {er }}$. Bien que son style soit différent de celui des manuscrits français de l'œuvre de Pigafetta, et ne peut donc être issu des mêmes ateliers, il témoigne lui aussi d'une mode pour les manuscrits cartographiques au début du XVI siècle.

39. Cf. notamment le manuscrit d'un collectionneur privé présenté dans M. Pelletier dir., Couleurs de la Terre, op. cit., p. 56. Cette hypothèse due à la seule comparaison visuelle mériterait une étude plus approfondie. 
cartographiques richement décorés. Antonio Pigafetta apparaît ainsi comme un auteur moyennement cultivé, qui met en avant l'authenticité de l'expérience directe et la description de peuples à l'apparence et aux mœurs exotiques pour transmettre ses impressions sur des terres entièrement nouvelles. Pour attirer l'attention de son public, il a commandé à une officine de libraires spécialisés dans les livres illustrés de cartes, des manuscrits de luxe réalisés sur le modèle des insulaires à la mode à l'époque. Par contraste, la lettre de Maximilianus Transylvanus apparaît comme l'ouvrage d'un érudit qui tente de mettre en perspective l'exploit de Magellan au regard du savoir antique.

\section{La lettre de Maximilianus Transylvanus}

La lettre en latin de Maximilianus Transylvanus, datée du 24 septembre 1522, est adressée au cardinal archevêque de Salzbourg, Matthäus Lang. Elle fut diffusée l'année suivante sous forme imprimée ${ }^{40}$, et précède donc la publication du témoignage d'Antonio Pigafetta. L'auteur n'a pas pris part à l'expédition : son analyse repose sur le récit oral des survivants de ce voyage, récit recueilli après le passage de la Victoria à Séville, le 8 septembre 1522. Le texte n'est pas illustré et ne contient pas ou peu d'informations maritimes. En revanche, les références au savoir de l'Antiquité y sont constantes, dans un rapport d'érudition et d'appel aux auctoritates de la géographie des humanistes. L'auteur cite en particulier Ptolémée et utilise les noms antiques tels que Chersonèse d'Or (Malacca), Cattigara, Taprobane, les Sères et les Scythes.

Or, ces références servent à mieux souligner la nouveauté radicale de l'exploit de Magellan et de ses compagnons. L'auteur est fier d'appartenir à une époque de progrès qui fonde son savoir sur l'expérience, par opposition à une connaissance uniquement théorique. En particulier dans l'introduction et la conclusion, il rappelle la teneur géographique et cosmographique du voyage, et souligne ce qu'il apporte en réfutant les erreurs du passé.

En effet tout ce que nous lisons chez les auteurs anciens, à propos des terres où naissent les épices, n'est que fable [...] je me contenterai de dire qu'Hérodote, auteur par ailleurs très illustre, raconte que l'on trouve la cannelle dans les nids de certains oiseaux, en particulier le Phénix [...] Pline, quant à lui, raconte que la cannelle pousse en Éthiopie près du pays des Troglodytes. Or l'on a maintenant découvert que la cannelle pousse bien loin de toute Éthiopie, car les nôtres, qui viennent de rentrer et ne connaissent rien mieux que l'Éthiopie, ont dû, avant de trouver ces îles et d'en revenir, faire le tour du monde, c'està-dire passer sous le très long parallèle plusieurs fois ${ }^{41}$.

40. Premières éditions à Cologne, Rome et Paris en 1523. Il existe treize éditions en latin ou en italien pour le seul Xvi ${ }^{\mathrm{e}} \mathrm{s}$. d'après X. DE CASTRO, op. cit., p. 884.

41. X. DE CASTRo, op. cit., p. 886. 
La période de la Renaissance est souvent présentée, de manière parfois un peu trop simple, comme une période de rupture avec le savoir antique et médiéval, grâce à l'apport des Grandes Découvertes. Or, la lettre de Maximilianus Transylvanus entretient justement un rapport ambigu à ce savoir antique, car, tout en saluant l'expérience nouvelle des navigateurs et la correction des erreurs du passé, il reconnaît par ailleurs que ces témoignages viennent au contraire valider certaines de ces antiques connaissances. Ainsi, certains peuples de la tradition antique et médiévale sont-ils considérés comme imaginaires :

Car qui pourrait croire que les Monoscèles, Sciapodes, Scyrites, Spitamées, Pygmées et bien d'autres créatures, plus monstrueuses qu'humaines, aient jamais existé ? ${ }^{42}$.

Mais une page plus loin, l'auteur cite comme des peuples réels les Ichtyophages et les Troglodytes rencontrés par les explorateurs portugais ${ }^{43}$.

La Géographie de Ptolémée sert par ailleurs de référence en ce qui concerne la mesure de la terre, au point que Maximilianus Transylvanus pense que les marins de Magellan se sont trompés en calculant leur position en longitude :

Comme les cosmographes anciens, et surtout Ptolémée, ont calculé 180 degrés de longitude depuis les îles Fortunées jusqu'à Cattigara vers l'est, nos marins, en naviguant le plus loin possible vers l'ouest à partir de ces îles Fortunées auraient dû parcourir 180 autres degrés jusqu'à Cattigara. Ils n'ont pu cependant effectuer ces mesures, ni placer, au cours d'une navigation si longue et si éloignée des terres, les marques et les bornes certaines de la longitude. Il me semble qu'en calculant ces longitudes ils se sont davantage trompés qu'autre chose, sans rapporter rien de certain. Cependant, quelle que soit la valeur de ces calculs, il ne faut pas les rejeter mais plutôt les admettre jusqu'à ce qu'on en trouve de plus sûrs ${ }^{44}$.

L'expérience des navigateurs devait donc, selon l'auteur, être étayée par des instruments scientifiques convaincants, et l'on sait l'importance qu'eut le problème du calcul de la longitude dans les explorations et la cartographie du $\mathrm{XVI}^{\mathrm{e}}$ et du XVII ${ }^{\mathrm{e}}$ siècle. À défaut, il fallait prendre en considération ces nouvelles données, tandis que les calculs du fameux cosmographe de l'Antiquité étaient considérés comme encore valables.

42. Ibid., p. 887.

43. Ibid., p. 887-888. La différence réside peut-être dans l'origine et la forme de ces peuples fabuleux. La première série est citée - encore dans les ouvrages de géographie du XVI ${ }^{\mathrm{e}}$ siècle d'après Pline et Solin, comme une humanité monstrueuse, déformée (un seul pied, une taille minuscule...) ; les Ichtyophages, mangeurs de poissons, et les Troglodytes, vivant dans des maisons souterraines, sont situés en Afrique d'après les cartes de Ptolémée. Ils ne se distinguent pas par leur apparence monstrueuse mais par leurs habitudes de vie. Cf. J. B. FrIEDMAN, The Monstrous Races in Medieval Art and Thought, Cambridge, 1981.

44. X. DE CASTro, op. cit., p. 892-893. 
Enfin, les références humanistes aux textes de l'Antiquité ne se cantonnent pas au domaine de la géographie, mais participent également de la rhétorique de l'éloge : l'auteur rend hommage aux navigateurs modernes qui ont surpassé les mythiques héros de la Toison d'Or :

Ces marins méritent davantage une gloire éternelle, assurément, que les Argonautes qui naviguèrent avec Jason jusqu'en Colchide. Leur navire luimême est encore plus digne d'être élevé parmi les étoiles que cette vieille Argo, qui a seulement navigué entre la Grèce et le Pont-Euxin, tandis que le nôtre, parti d'Hispalis en direction du midi, a traversé tout l'occident, est passé en bas de notre hémisphère puis a pénétré en orient, pour revenir ensuite en occident ${ }^{45}$.

D'une certaine manière, le texte de Maximilianus Transylvanus fait ici écho à la culture humaniste d'un Buondelmonti, évoquant sans cesse les navigations des anciens Grecs en Méditerranée. La découverte des îles du Nouveau Monde vient ainsi rejoindre et compléter la description des îles du monde antique.

La comparaison du témoignage d'Antonio Pigafetta et de la lettre de Maximilianus Transylvanus fournit deux axes de réflexion. D'une part, elle permet de souligner combien la connaissance approfondie du savoir antique, acquise par les travaux humanistes, a permis de mieux comprendre le véritable impact des Grandes Découvertes. C'est parce que Maximilianus Transylvanus et ses lecteurs savent l'apport et les limites de Ptolémée, qu'ils peuvent mesurer ce que l'expérience nouvelle des navigateurs apporte à la connaissance du monde. D'autre part, ces deux sources montrent à quel point la mise en forme littéraire et artistique du récit était importante pour rendre compte des Découvertes, pour faire admettre au public cultivé de l'époque la nouveauté radicale des explorations de Magellan et de ses compagnons. Humanisme et géographie à la Renaissance sont intimement liés parce que les travaux des humanistes ont fourni le cadre de réflexion nécessaire aux Grandes Découvertes et à leur interprétation. C'est aussi l'essor de l'industrie du livre, manuscrits cartographiques et imprimerie développés par les humanistes, qui a fourni le cadre éditorial de diffusion des Grandes Découvertes. Les récits de voyage vers le Nouveau Monde n'ont pas seulement fait l'objet d'un compte rendu brut d'une nouvelle expérience, mais ont été mis en forme pour être correctement compris et interprétés, afin de mieux lire et corriger le savoir passé.

Emmanuelle VAGnon - Docteur en histoire médiévale, Université Paris I, evagnon@yahoo.fr 


\section{De la Grèce antique au voyage de Magellan. Les modèles humanistes d'Antonio Pigafetta et de Maximilianus Transylvanus}

Le voyage de Fernand de Magellan et de ses marins est connu par plusieurs sources différentes, dont le témoignage direct d'Antonio Pigafetta (1523) et la lettre de Maximilianus Transylvanus, secrétaire de Charles Quint (1522). Ces deux exemples montrent à quel point ces récits d'un des épisodes les plus remarquables des Grandes Découvertes s'inscrivent dans un rapport ambigu de rupture mais aussi d'hommage par rapport au savoir humaniste, tout en requérant les supports éditoriaux conformes aux goûts des cercles cultivés de l'époque. Les manuscrits de Pigafetta rappellent la forme de l'isolario, genre cartographique inauguré par l'œuvre de l'humaniste florentin Cristoforo Buondelmonti au $\mathrm{XV}^{\mathrm{e}}$ siècle, et très imité au début $\mathrm{du}_{\mathrm{XVI}}{ }^{\mathrm{e}}$ siècle. Maximilianus Transylvanus utilise des références à la géographie de l'Antiquité pour souligner l'apport des nouvelles découvertes.

récit de voyage - grandes découvertes - isolario - cartes - humanisme

\section{From Ancient Greece to Magellan's Travel:}

\section{Humanistic Models of Antonio Pigafetta and Maximilianus Transylvanus}

The paper considers two accounts of Magellan's travel around the world: the Navigation \& découvrement de l'Inde supérieure \& îles de Malucque, by Antonio Pigafetta (from manuscripts of 1523-1525), and a Letter written by Maximilianus Transylvanus, dated 1522. It evaluates the humanistic practices and knowledge lying at the foundations of these works: the pictural and cartographic model of the isolario used by Pigafetta, the critical references to Ancient geography by Maximilianus Transylvanus.

travel narrative - great discoveries - isolario - maps - humanism 